\title{
Improving Medication Adherence with Two-way Short Message Service Reminders in Sickle Cell Disease and Asthma
}

\section{A feasibility randomized controlled trial}

Brandi M. Pernell1,2; Michael R. DeBaun; Kathleen Becker; Mark Rodeghier ${ }^{4}$; Valencia Bryant ${ }^{1}$; Robert M. Cronin ${ }^{5}$

${ }^{1}$ Vanderbilt-Meharry-Matthew Walker Center of Excellence in Sickle Cell Disease, Vanderbilt University Medical Center, Nashville TN;

2University of Alabama at Birmingham, Department of Pediatrics, Division of Hematology, Birmingham, AL;

3Johns Hopkins University, School of Nursing, Baltimore, MD;

${ }^{4}$ Rodeghier Consultants, Chicago, IL;

${ }^{5}$ Vanderbilt University Medical Center, Departments of Biomedical Informatics, Internal Medicine, and Pediatrics, Nashville, TN

\section{Keywords \\ Sickle cell anemia, asthma, medication adherence, short message service, text}

\section{Summary}

Introduction: Sickle cell disease (SCD) is a childhood and adult disease that primarily affects African Americans, characterized by life threatening sequelae mitigated by medications. One-way and two-way short message service (SMS) medication reminders have differing efficacy in chronic diseases. There is limited literature about SMS medication reminders in SCD.

Objective: The goal of this study was to test the feasibility, defined by recruitment/acceptance, retention/attrition, and technology utilization, of two-way SMS medication reminders in individuals with SCD with and without asthma.

Materials and Methods: Participants were randomly allocated to standard care or reminders. Two-way SMS reminders were automated using Research Electronic Data Capture (REDCap) for hydroxyurea, fluticasone, budesonide and montelukast. Adherence was measured using the Morisky Medication Adherence Scale-8 (MMAS-8). Asthma control was assessed using the Childhood and Adult-Asthma Control Tests (ACT). Participants were enrolled 28 to 60 days with a common termination date.

Results: The recruitment rate was 95\% (47/49) and $82.9 \%$ completed the study. Among the 47 study participants enrolled, $51.1 \%$ were male, $61.7 \%$ were adults, median age was 20 (range: 3 to 59 ), and $98 \%$ were African Americans. Of the 26 participants receiving messages, $20 \%$ responded on over $95 \%$ of the days and usage varied with an average response rate of $33 \%$, ranging from $21 \%$ to $46 \%$. Medication adherence scores improved significantly in the intervention group (3.42 before, 5.46 after; $p=0.002$ ), but not in the control group ( 3.90 before, 4.75 after; $p=0.080$ ). Childhood-ACT scores improved in the intervention group (19.20 before, 24.25 after). Adult-ACT scores within the intervention arm were unchanged (21.0 before, 22.0 after. ACT scores did not improve significantly.

Conclusion: This study demonstrated the feasibility for two-way SMS medication reminders to improve medication adherence in a high-risk population where daily medication adherence is critical to health outcomes and quality of life. 


\section{Correspondence to:}

Robert M. Cronin

Department of Biomedical Informatics, Vanderbilt University Medical Center,

2525 West End Suite 1475,

Nashville, TN 37203, USA

Email: robert.cronin@ vanderbilt.edu
Appl Clin Inform 2017; 8: 541-559

https://doi.org/10.4338/ACI-2016-12-RA-0203

received: December 4, 2016

accepted: March 8, 2017

published: May 24, 2017

Citation: Pernell BM, DeBaun MR, Becker K, Rodeghier M, Bryant V, Cronin RM. Improving medication adherence with two-way short message service reminders in sickle cell disease and asthma: A feasibility randomized controlled trial. Appl Clin Inform 2017; 8: 541-559

https://doi.org/10.4338/ACI-2016-12-RA-0203

Funding

This work was supported by the Junior League of Nashville, HRSA grant number

5-U38-MC2222-0-04-00, and the Trans-Institutional Programs at Vanderbilt University Medical Center. 


\section{Background and Significance}

Sickle cell disease (SCD) is an inherited red blood cell (RBC) disorder, characterized by early RBC destruction, vessel occlusion and compromised end-organ perfusion. In the United States, there are an estimated 100,000 individuals who have with SCD, who are primarily African American [1]. Advancements in the care of individuals with SCD have led to improved life expectancy. Disease knowledge and effective self-management are critical to the advancement of SCD care. Health information technology has evolved as a one of the leading catalyst for increased health care knowledge among consumers.

Health information is one of the leading topics researched by internet users. While the most likely groups to search for online health information are Caucasians, women and individuals aged 18-49 years, younger and minority populations are more likely to use mobile devices to gather information, lending mobile health technology as a potential strategy for improving access and utilization of electronic health information. [2] The use of mobile health technology to improve health outcomes has been demonstrated in other chronic disease populations. However, there are large gaps in the literature regarding the use of technology by the SCD population [3], as well as widely-known health care disparities and disparities in health information technology adoption among African Americans [4-7].

Vaso-occlusive pain is the most common complication affecting individuals with SCD, followed by acute chest syndrome (ACS), which is an acute pulmonary condition. Hydroxyurea, which increases the synthesis of fetal hemoglobin and raises the total hemoglobin, results in decreased red blood cell sickling, abating the frequency of vaso-occlusive pain and ACS [8-10]. In addition to hydroxyurea therapy, early detection and management of asthma is recommended given the associated risk of increased vaso-occlusive pain rates and ACS among individuals with SCD and concomitant asthma [11, 12]. The National Asthma Education Prevention Program-Expert Panel Report 3 (NAEPP-EPR 3) provides evidence-based guidelines for the detection and management of asthma and recommends inhaled corticosteroids as the cornerstone treatment for persistent asthma symptoms [13]. Both inhaled corticosteroids and hydroxyurea therapy require daily adherence to achieve treatment effectiveness.

Daily medication adherence is less than optimal for individuals with SCD, with non-adherence rates reported as high as $40 \%$ in individuals with SCD, compared to an average non-adherence rate of $24.5 \%$ in other chronic disease populations [14-17]. Adherence in SCD is associated with important clinical outcomes including reduced risk of SCD-related hospitalization, all-cause and SCD-related emergency department visits, vaso-occlusive events, and health related quality of life [14, 18]. Short message service (SMS) medication reminders are an emerging technology employed to facilitate behavioral change and decrease medication non-adherence. Johnson et al. observed improved medication adherence and satisfaction with mobile technology but lower usage in African Americans when compared to Whites. [19] A web based application was used and required registration before receiving SMS text messages. We believe adherence to the use of the technology and medication adherence should improve because of increased use of patient portals in the general population $[5,20]$ and the pervasiveness of mobile technologies [21-24]. A recent pooled meta-analysis demonstrated improvement in medication adherence with SMS reminders among individuals with chronic illnesses [25]. However, the text messaging methodologies from the studies compared in the meta-analysis included both one-way and two-way messaging, with some studies showing no improvement in outcomes and no clear delineation of an optimal SMS reminder strategy. A potential improvement in SMS communication is the use of two-way SMS communication versus one-way communication. In one-way SMS communication, a text message is sent to a recipient with no allowance or expectation for a response, while two-way SMS communication permits a recipient response. While this recent pooled meta-analysis [26] did not demonstrate whether one-way or twoway messaging is superior, a more recent meta-analysis demonstrated that two-way SMS messages are superior in improving medication adherence as compared to one-way [27]. The perceived advantage of two-way SMS communication is the opportunity for patients and families to demonstrate self-management behaviors prompted by the SMS communication. Further, two-way SMS communication produces subjective adherence data, which health care providers can utilize during clini- 
cal encounters to provide positive feedback for high adherence, or explore barriers to medication compliance and suggest alternative strategies to improve low adherence rates.

Despite the wide access to mobile technologies [21-24] and that SMS reminders have been shown to produce significant improvement in medication adherence in individuals with various illnesses [25], very few studies have examined SMS medication reminders in children or adults with SCD. In a retrospective study of one-way SMS reminders, researchers at St. Jude Children's Research Hospital demonstrated improved medication adherence in children with SCD through medication possession ratio and biomarkers [26]. While this study showed improved medication adherence with SMS reminders, the study was limited by a retrospective evaluation of adherence to a single medication through a one-way text messaging intervention for children with SCD. The purpose of this prospective, randomized controlled pilot study was to test the feasibility of two-way SMS medication reminders and to evaluate the impact on adherence to one or multiple medications and asthma symptom control among children and adults with SCD or SCD and concomitant asthma. In a prospective feasibility randomized controlled trial, in children and adults with SCD, we tested the hypothesis that daily two-way SMS medication reminders over a 60-day period is an acceptable intervention that can improve adherence to multiple medications and improve asthma symptom control.

\section{Methods}

This study was approved by the Vanderbilt University Medical Center Institutional Review Board. The randomized feasibility trial used a parallel study design with equal allocation in permuted blocks of 10 participants using GraphPad [28]. Study participants were de-identified and blinded from one of the coauthors who randomly assigned participants to either the intervention or control group based on GraphPad numbering. Feasibility, the primary outcome, was defined by recruitment rate (acceptance rate), retention rate (1-attrition rate), adherence with the technology (or utilization of the technology). The secondary outcomes included medication adherence and asthma control.

\section{Setting}

Study recruitment took place in the Vanderbilt-Meharry Center of Excellence of Sickle Cell Disease, located in Nashville, TN. Medical care is delivered in one pediatric and one adult outpatient hematology clinic, located in a tertiary academic medical center as well as a community-based, integrated pediatric SCD and asthma evening and weekend clinic located in a community health center in Nashville, TN.

\section{Study participants}

Study eligibility criteria included: ages 1-70 years, a confirmed diagnosis of SCD based on hemoglobin analysis \pm a diagnosis of mild, moderate, or severe persistent asthma with prescribed asthma control therapy and/or hydroxyurea therapy. Given the time to treatment effect, participants with SCD and asthma, who were prescribed daily asthma control therapy (such as fluticasone, montelukast or budesonide) and/or hydroxyurea for a minimum of three months prior to study enrollment, were eligible for participation. Study participants were randomly allocated to either the treatment arm (daily two-way SMS medication reminders) or control arm (standard care), stratified by age categories: adult ( $\geq 18$ years) and pediatric ( $<18$ years). Participants received SMS messages on their own cell phones. Participants were enrolled for a minimum follow up period of at least 28 days and up to 60 days based on a common termination date for all participants.

\section{Intervention}

SMS medication reminders were sent over a 60-day period using Research Electronic Data Capture (REDCap) software, a web-based application designed to support survey development, electronic data capture, and exportation into statistical software for analysis [29]. Daily and twice daily, auto- 
mated surveys were manually set up in REDCap by two co-authors (BM and RC) based on prescribed medication regimens. This was accomplished through the automated invitations feature in REDCap ( Figure 1). Automated surveys were manually set up for each of the three arms of the study (asthma, hydroxyurea, both asthma and hydroxyurea) for all 60 days. Study participants provided individualized preferences regarding time of day to receive daily SMS medication reminders based on the times of day they took their hydroxyurea or asthma medications. The SMS reminders included the following text: "Did you take your [hydroxyurea] [asthma] medication [this morning] [this evening] [today]?" If the participant was prescribed both hydroxyurea and asthma medications, a separate text message was sent for each of the medications. Participants taking hydroxyurea alone received one text message at the time of day they specified. Participants taking twice-daily asthma medications received two text messages at two separate times of the day, once in the morning at a time they specified, and one 10 hours later (to mimic the twice per day medication schedule). If the participants took both hydroxyurea and asthma medications, they received two text messages at two separate times of the day, once in the morning at a time they specified (for the morning asthma medication), and one 10 hours later asking if they took both their asthma medication and hydroxyurea. Study participants receiving SMS medication reminders were prompted to reply '(1) yes' or '(0) no' as to whether they took their medication. Participants that responded ' $(0)$ no' received an additional SMS reminder two hours later reminding them to take their medication. If the participant did not respond to the initial text message, one additional text reminder with the same message was sent one hour later. Study participant responses were captured through REDCap. Monitoring of the study participants' responses to daily SMS medication reminders in REDCap was conducted weekly and study updates and reminders about the study were provided during regularly scheduled clinic encounters to maintain participant engagement.

\section{Sample size calculation}

Given the intent of this study to assess feasibility, a sample size large enough for power to test the null hypothesis was not needed [30]. However, a sample size of at least 30, based on the central limit theorem, with an anticipated attrition rate of $20 \%$ was used to set the minimum sample size for this study. Although not needed for this feasibility study, a sample size of 46 (23 participants in each treatment group) with $80 \%$ power would be needed to detect a clinically significant change in baseline Asthma Control Test (ACT) scores and the ACT threshold for well-controlled asthma of 23 and 22 for adults and children, respectively.

\section{Outcome measures}

\section{Recruitment rate}

Study eligibility was determined through medical record review and confirmation of inclusion and exclusion criteria. Children and adults with SCD and asthma eligible for study participation were approached during routine clinic encounters over a six-month timespan, starting in September of 2015. The recruitment rate was defined as the proportion of eligible participants approached who enrolled in the study.

\section{Retention rate}

Study participants completing both the pre-intervention and post-intervention data collection phases were designated as completing the study. The retention rate was defined as the proportion of enrolled participants that completed the study.

\section{Morisky Medication Adherence Scale Scores}

The Morisky Medication Adherence Scale-8 (MMAS-8) is a validated, 8-item, self-report tool designed to capture self-reported adherence to prescribed medication regimens and circumstances surrounding adherence behavior ( Figure 2) [31]. This scale has been tested in multiple chronic diseases, including individuals with SCD [16, 31-33]. Baseline adherence scores were captured immediately following study enrollment and after the intervention phase. Parents completed the scale for children participants younger than 18. Adult participants over 18 completed their own scale. 
Medication adherence scores range from $0-8$, with a score $<6$ indicative of low medication adherence.

\section{Asthma Control Test and TRACK Scores}

The Asthma Control Test (ACT) is a validated, patient-based tool used to quantify patients' and caregivers' perceptions of asthma symptom control [34]. Study participants, 4-11 years of age, with asthma used the Childhood-ACT scoring tool ( $>$ Figure 3 ) and those $\geq 12$ years of age with asthma used the Adult-ACT scoring tool ( $\$$ Figure 4). The Test for Respiratory and Asthma Control in Kids (TRACK) is a validated, patient-based tool designed to assess caregivers' perceptions of their children's asthma symptoms, and was administered to the parents and caregivers of study participants $<=5$ years of age with asthma ( $>$ Figure 5 ). Baseline ACT and TRACK scores were captured immediately following study enrollment. Post-intervention ACT and TRACK scores were assessed at the conclusion of the study phase. Childhood and Adult-ACT scores $\leq 19$ and TRACK scores $<80$ were indicative of uncontrolled asthma [35]. A Childhood-ACT score $>22$ and Adult-ACT score $>23$ indicated well-controlled asthma. Participant ACT and TRACK scores were analyzed as continuous and binary variables (controlled and uncontrolled). A 2-point change in Childhood-ACT and a 3-point change in Adult-ACT were considered a clinically meaningful improvement in asthma symptom control $[35,36]$. A change in TRACK score of 10 or more was considered a clinically meaningful change in asthma control [37].

\section{Statistical analysis}

Participant demographics were examined using descriptive statistics. Categorical variables were reported as frequencies and differences between groups assessed with a chi-square test. Recruitment and retention were calculated as proportions. Pre and post mean medication adherence and changes in adherence within and between treatment groups were analyzed using a mixed methods model to account for missing data and correlations between patient scores. Statistical differences among groups in ACT scores are not reported because of the limited number of participants in the combined groups defined by control/treatment and child/adult categories. We performed sub-analyses comparing the change in medication adherence between the control and intervention groups of adults, children, asthma medications alone and hydroxyurea alone. Statistical significance was defined as a $\mathrm{p}$ value of $<0.05$.

\section{Results}

\section{Participant characteristics}

Among the 47 study participants enrolled, $51.1 \%$ were male, $61.7 \%$ were adults, median age was 20 (range: 3 to 59), and 98\% were African Americans. The majority, 89.3\% of study participants had hemoglobin SS SCD phenotype and were on hydroxyurea therapy. A comorbid diagnosis of asthma was present in $57.4 \%$ of the study participants. There were 26 study participants randomized to the intervention group and 21 in the control group. No statistically significant differences were noted in participant characteristics among the intervention and control groups at baseline ( Table 1 ).

\section{Retention and recruitment rates}

The recruitment rate for this feasibility trial was exceptionally high. There were 49 children and adults with SCD and asthma approached for study participation, of which $95.9 \%$ agreed to study participation. Of the 47 participants enrolled, $82.9 \%$ completed the study. Retention rates were not significantly different between the control and intervention groups $(\mathrm{p}=0.217)$. 


\section{Variation in proportion of short message reminder participant response}

The response to the two-way SMS prompt was mixed; a small group responded to almost every prompt and an equally small group did not respond to any prompts. Of the 26 participants that were allocated to the intervention arm, one did not receive text messages due to an error with the technology. Eight of the remaining 25 (32\%) never responded to any text messages. Twenty percent of participants responded on more than $95 \%$ of the days that text messages were sent. Usage varied depending on the different days with an average response rate of $33 \%$, ranging from $21 \%$ to $46 \%$ ( Figure 6). Partial responders, those that responded at least once but less than $95 \%$ of the time, had similar variation as the overall responders ( $>$ Figure 7). A total of 669 text messages were responded to, with 53 responding 'no' indicating the respondents did not take their medication. There were 35 responses to the second reminder text. Of these second reminders, 22 responded 'yes' meaning that they took their medications after receiving a second reminder text. All of the second reminder responses of 'no', indicating that they did not take their medication after two reminders, came from two participants ( Table 2). Participants that received once daily text message reminders had a higher percentage of days that they responded (median: $21.7 \%$, IQR: $0.0,83.3 \%$ ) than those who received twice daily text message reminders (median:3.3\%, IQR: 0.0,85.0\%), however this difference was not significant $(\mathrm{p}=0.536)$.

\section{Short message service reminders improve medication adherence scores}

Baseline and endpoint medication adherence rates were not statistically different between the two groups ( $>$ Table 1 ). The intervention group showed a statistically significant improvement in medication adherence scores (3.42 before, 5.46 after; $p=0.001$ ), whereas the control group did not (3.90 before, 4.75 after, $\mathrm{p}=0.080$ ) The increase (improvement) on the MMAS- 8 was not significantly different between the two groups ( $\mathrm{p}=0.118$, $>$ Table 3 ). Our sub-analysis demonstrated a greater increase in adherence in the intervention group compared to the control group in those with asthma medications alone, and of children alone, but the differences were not statistically significant (asthma medications alone: $\mathrm{p}=0.182$; children: $\mathrm{p}=0.156$ ).

\section{Medication reminders demonstrate a clinically significant improvement in asthma control}

Self-reported asthma symptom control improved clinically with the use of SMS medication reminders. Among the 27 study participants with SCD and concomitant asthma, 2 were $<4$ years of age, requiring the TRACK assessment tool, and were thus excluded from the analysis. Post-intervention Childhood and Adult-ACT scores for study participants $\geq 4$ years of age improved within both the intervention and control groups. Among study participants, 4-11 years of age, Childhood-ACT scores improved by a raw score of 5.1 points (19.2 before, 24.3 after) in the intervention group and 2.4 points (18.8 before, 21.2 after) in the control group. Pre and post Adult-ACT scores for the intervention group were 21.0 and 22.0, respectively. Pre and post Adult-ACT scores for the control group were 21.67 and 22.67 , respectively.

\section{Discussion}

Medication adherence is critical to effective chronic disease self-management. Children and adults with SCD or SCD and concomitant asthma represent high-risk populations in which daily medication adherence is critical to health outcomes and quality of life. Perceived medication adherence has been shown to be an accurate surrogate for measuring adherence to hydroxyurea in SCD. [16, 18] In this pilot randomized controlled trial we evaluated the feasibility of two-way SMS medication reminders for children and adults with SCD and asthma by measuring recruitment and retention rates, and technology adherence (utilization). We also measured medication adherence, and overall asthma control. Our results demonstrated improved medication adherence, as well as high recruit- 
ment and retention rates, with the use of SMS medication reminders, deeming SMS reminders as a feasible and promising intervention for children and adults with SCD and asthma.

Recruitment and retention rates were used to assess the feasibility of two-way SMS communication in this SCD cohort. A previous trial examining the efficacy of SMS medication reminders for children with SCD was done retrospectively; therefore, the feasibility of conducting a prospective trial among individuals with SCD that involved text messaging to their mobile phones was not previously known [26]. We achieved excellent recruitment (96\%) and retention rates (83\%) demonstrating the feasibility of this trial, with higher levels than the previous retrospective study that had $91 \%$ recruitment and $77 \%$ retention. Participants received a gift card incentive for filling out the survey on the first research visit (\$15) and the final research visit (\$15). No incentives were given for technology use. Although there was a small incentive to participate in the study, we postulate that the high recruitment and retention rates were attributable to the presentation of SMS medication reminders as a way for patients and families to becomes partners in health and take on more ownership for improved health outcomes.

Medication adherence improved with the use of daily, two-way SMS medication reminders in the intervention group but not in the control group. SMS text messaging has been used successfully to deliver medication adherence reminders; however, two-way text messaging has not always demonstrated improved medication adherence [38-40]. Estepp et al. demonstrated improved adherence to hydroxyurea using one-way text messaging in a retrospective trial in children with SCD [26]. Their study demonstrated improved medication possession ratios using one-way text messaging in children for hydroxyurea, and we found that two-way text messaging improved self-reported medication adherence using the MMAS- 8 in adults and children with SCD. Our study adds to this preexisting literature indicating that SMS reminders are an effective strategy for improving self-reported medication adherence in children and adults with SCD. A sub-analysis of adults, children, asthma medication alone, and hydroxyurea alone demonstrated improvement in medication adherence but without statistical significance. The change in adherence was higher in children and in those using asthma medications alone.

While this feasibility trial was not designed to assess clinical improvement in ACT scores, we did observe an improvement in ACT scores in the intervention group. Two SMS messaging studies involving individuals with asthma looked primarily at one-way text messaging [41, 42]. In both of these studies, medication adherence was significantly improved by SMS messaging, as demonstrated in our study. Lv et al. observed a significant improvement in subjective measures, using the Perceived Control of Asthma Questionnaire, PCAQ-6) and quality of life with SMS messaging; however, neither of these studies demonstrated significant improvement in clinical outcomes such as FEV1\% or emergency room visits for asthma in the SMS group as compared to the control group. Our study did show a trend towards significant change in ACT scores and a larger sample size may demonstrate similar findings of improved asthma control as other studies.

Participant response rates varied. Participants fell into one of three groups: non-responders who never responded, partial responders, and usual responders who responded on more than $95 \%$ of the days. The most common participant type was partial responders. About one third of all participants responded to the text message reminders on any given day, whereas other studies have reported $22-100 \%$ response rates [43]. Johnson et al. demonstrated that most of the African American participants never used their text messaging intervention [19], whereas $98 \%$ of our participants were African American with the majority responding at least once. This could be related to patient engagement as all participants are from a sickle cell center where providers and healthcare teams actively engage their patients to know their disease and medications. The providers also promote technology to help with patient care.

Further research evaluating why participants do not respond could yield better ways to improve adherence to two-way text messaging responses in the SCD population. Potential reasons to account for why individuals do not respond to text message reminders include changing phone numbers without notifying the research team $[44,45]$, failing to respond because they find the messages intrusive,[41] finding the messages disruptive to their daily routines [42, 46], and changing their behavior from using the system for a short period of time and no longer feeling the need to reply [19, 42]. There may be features to the technology that could be used to address these different reasons including personalization of messages to decrease intrusiveness, allowing participants to modify settings 
so messages are not disrupting their daily routines, or automatically notifying the research team if there is no response after a few days. While the researchers received anecdotal information about why users did not use the system including the fact they did not know the reminders were from the research team and they changed phones before receiving messages, this study did not explore the qualitative reasons why some participants responded more often than others, which is an area for further research. While statistically significant differences were not observed, we also found that response rates were higher in participants that received only a single message per day as compared to multiple messages per day. These response rate differences could demonstrate the poor adherence to the technology due to the burden of having to respond to message multiple times per day. We observed an improvement in medication adherence in the intervention group when compared to the control group, indicating that study participants' behavior changed irrespective of response rates.

Participant responses decreased with time. The absence of a response to the text message did not appear to impair medication adherence, indicating that initial SMS messaging may have prompted habit formation which no longer required rapid-cycle reinforcement. While our study demonstrated that two-way text messaging improved medication adherence for individuals with SCD, asthma alone (without SCD), or both over a 30-day period, further research over longer periods of time is needed to determine the necessity for persistent responses and the effect of the text messaging system on medication adherence for prolonged periods. Also, even if participants did not respond, all participants continued to receive text messages. Quite possibly the ongoing text messaging served as a prompt sufficient enough for participants to initiate taking their medication, but the participants were not sufficiently motivated to respond. Further research is needed to determine if improved medication adherence is due to daily two-way text message reminders regardless of the participant's response.

The use of the second reminder varied and may be useful in determining who might need further interventions to improve medication adherence. Most responses to the initial reminder indicated that participants remembered to take their medication ( $92 \%$ responded 'yes' to the first reminder). Of the 'no' responses to the first reminder, about $42 \%$ of the second reminders had a 'yes' response, meaning the respondents remembered to take their medication after forgetting initially and receiving a second reminder. Only eight of the participants used this feature at least once, likely indicating that while this feature was not widely used, it was helpful in reminding some participants. Another advantage of the two-way text messaging system is that health care team members can monitor how well individuals with SCD take their medications based on self-report. In this study, there were two participants who missed doses after two reminder messages, indicating the need for additional interventions by the health care team.

\section{Limitations}

This study had several limitations. The intervention phase was of short duration, ranging from a minimum of 28 days to a maximum of 60 days, leaving room for further demonstration of treatment effects of SMS medication reminders in regards to asthma symptom control and SCD-related morbidity. Previous literature on the use of SMS reminders in SCD demonstrated sustained adherence effects lasting up to 12 months using SMS reminders for medication adherence [26]. This study had a $17.1 \%$ attrition rate, potentially diminishing the effect of SMS medication reminders. This study did not compare the difference in child versus caregiver receipt of SMS medication reminders. Further research in reporting medication possession ratios through publicly available databases and clinical outcome data would decrease this bias. This study used the MMAS- 8 to measure medication adherence and did not evaluate lab markers of adherence, such as mean corpuscular volume $(\mathrm{MCV})$. While a perceived medication adherence score may have recall bias, the MMAS- 8 has been shown to be an accurate surrogate for measuring adherence to hydroxyurea in SCD [18]. This study did not measure important clinical events, such as ER visits or hospitalizations. Further research in larger cohorts could help determine if text messaging reminders have effect on clinical outcomes. No behavior change theory was used to design or inform the study which could limit the findings. Finally, the study was done as a small feasibility trial at a single institution of a convenience sample of patients, therefore the results cannot be generalized to a larger study population. 


\section{Conclusion}

Two-way SMS medication reminders are feasible in children and adults with SCD and asthma, and could provide more information to health care providers about how to improve medication adherence. The two-way SMS medication reminders are a low-tech solution that can improve self-management in a crucial vulnerable population. Larger, multicenter studies of longer durations are needed to further examine the health-related implications of SMS medication reminders in children and adults with SCD and asthma, including objective measures of asthma symptom control, rates of vaso-occlusive pain and ACS, quality of life, and mortality. The findings from this study lay the foundation for future studies examining this novel approach designed to improve medication adherence in individuals with SCD and asthma.

\section{Abbreviations}

- SCD: sickle cell disease

- RBC: red blood cell

- ACS: acute chest syndrome

- NAEPP-EPR 3: National Asthma Education and Prevention Program, Expert Panel Report 3

- SMS: short message service

- REDCap: Research Electronic Data Capture

- ACT: Asthma Control Test

- TRACK: Test for Respiratory and Asthma Control in Kids

- PCAQ-6: Perceived Control of Asthma Questionnaire

- FEV1: forced expiratory volume in the first second

\section{Clinical Relevance Statement}

Medication adherence is critical to effective chronic disease self-management. Individuals with sickle cell disease are high-risk populations in which daily medication adherence is critical to health outcomes and quality of life. In this manuscript, we demonstrate through a pilot randomized clinical trial that mobile applications, such as two-way SMS messaging, can improve medication adherence in individuals with SCD.

\section{Multiple choice questions}

Which of the following is true of using Short Message Service (SMS) text messaging for medication adherence?

A) Two-way SMS messaging is always superior to one-way text messaging for medication adherence

B) Two-way SMS messaging improves long-term medication adherence in sickle cell disease

C) Respondents to two-way SMS messaging fall into two categories, those that use it every day, and those that never use it

D)Large randomized trials are needed to demonstrate that SMS medication reminders can improve clinical outcomes

E) Most users never respond to two-way SMS medication reminders

Answer: D

\section{Conflicts of Interest}

There are no potential conflicts of interests among the listed authors.

\section{Human Subjects Protection}

This study was approved by the Vanderbilt University Medical Center Institutional Review Board 


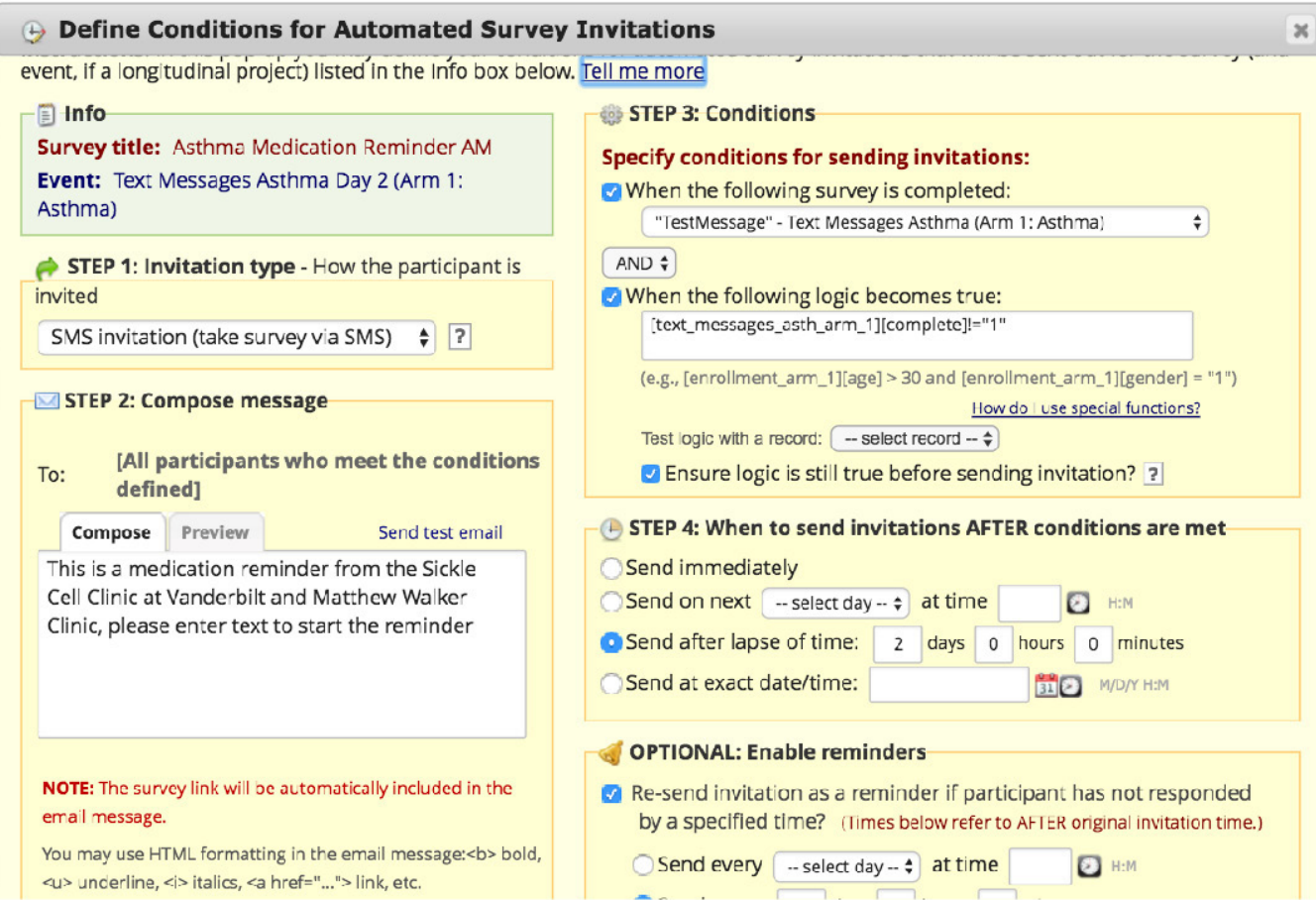

Fig. 1 Screenshot of an example of a Research Electronic Capture Database (REDCap) automated survey invitation.

1.Do you sometimes forget to take your pills?

2.People sometimes miss taking their medication for reasons other than forgetting. Thinking over the past two weeks, were there any days when you did not take your medicine?

3. Have you ever cut back or stopped taking your medicine without telling your doctor because you felt worse when you took it?

4.When you travel or leave home, do you sometimes forget to bring your medicine with you?

5.Did you take your medicine yesterday?

6.When you feel like your health is under control, do you sometimes stop taking your medicine?

7.Taking medication every day is a real inconvenience for some people. Do you ever feel hassled about sticking to your treatment plan?

8. How often do you have difficulty remembering to take your medicine?

$\square$ Never/rarely

$\square$ Once in a while

口Sometimes

$\square$ Usually

$\square$ All of the time

Fig. 2 Morisky Medication Adherence Scale. Self-report tool designed to capture medication adherence behaviors. Each 'no' $=1$, with the exception of number 5 , where 'yes' $=1$. Item 8 is scored as follows: Never/Rarely $=1$, everything else $=0$. A score of $\geq 8=$ high adherence; 6 to $<8=$ moderate adherence; $<6=$ low adherence. 


\section{Childhood Asthma Control Test for Children 4 to 11 Years}

Have your child complete these questions.

1. How is your asthma today?
0 Very bad
1 Bad
2 Good
3 Very good

SCORE $=$

2. How much of a problem is your asthma when you run, exercise or play sports?

0 It's a big problem, I can't do what I want to do

1 It's a problem and I don't like it

2 It's a little problem but it's okay

3 It's not a problem

SCORE $=$

3. Do you cough because of your asthma?

0 Yes, all of the time

1 Yes, most of the time

2 Yes, some of the time

3 No, none of the time SCORE =

4. Do you wake up during the night because of your asthma?

0 Yes, all of the time

1 Yes, most of the time

2 Yes, some of the time

3 No, none of the time

SCORE $=$

Please complete the following questions on your own.

1 During the last 4 weeks, how many days did your child have any daytime asthma symptoms?

Not at all $=5 \quad 1-3$ days $=4 \quad 4-10$ days $=3 \quad 11-18$ days $=2 \quad 19-24$ days $=1$ Everyday $=0$ SCORE $=$

2 During the last 4 weeks, how many days did your child wheeze during the day because of asthma?

Not at all $=5 \quad 1-3$ days $=4 \quad 4-10$ days $=3 \quad 11-18$ days $=2 \quad 19-24$ days $=1$ Everyday $=0$

SCORE $=$

3 During the last 4 weeks, how many days did your child wake up during the night because of asthma?

Not at all $=5 \quad 1-3$ days $=4 \quad 4-10$ days $=3 \quad 11-18$ days $=2 \quad 19-24$ days $=1 \quad$ Everyday $=0$ SCORE $=$

TOTAL =

Fig. 3 Percent of Participants Responding to Text Messages. Childhood Asthma Control Test. Patient and caregiver self-report tool of asthma symptoms in children 4-11 years of age. A score $\leq 19$ indicates uncontrolled asthma. 


\section{Asthma Control Test (ACT) for People 12 Years \& Older}

1. In the past 4 weeks, how much of the time did your asthma keep you from getting as much done as work, school or home?
1 All of the time
2 Most of the time
3 Some of the time
4 A little of the time
5 None of the time

SCORE $=$

2. During the past 4 weeks, how often have you had shortness of breath?

1 More than once a day

2 Once a day

33 to 6 times a week

4 Once or twice a week

5 Not at all

SCORE $=$

3. During the past 4 weeks, how often did your asthma symptoms (wheezing, coughing, shortness of breath, chest tightness or pain) wake you up at night or earlier than usual in the morning?

14 or more nights a week

22 or 3 nights a week

3 Once a week

4 Once or twice

5 Not at all

SCORE $=$

4. During the past 4 weeks, how often have you used your rescue inhaler or nebulizer medication (such as albuterol)?

13 or more times per day

21 or 2 times per day

32 or 3 times per week

4 Once a week or less

5 Not at all

SCORE $=$

5. How would you rate your asthma control during the past $\mathbf{4}$ weeks?
1 Not controlled at al
2 Poorly controlled
3 Somewhat controlled
4 Well controlled
5 Completely controlled

SCORE $=$

TOTAL

Fig. 4 Adult Asthma Control Test. Patient self-report tool of asthma symptoms for individuals $\geq 12$ years of age. A score $\leq 19$ indicates uncontrolled asthma. 


\section{TRACK - Test for Respiratory \& Asthma Control in Kids}

1. During the past 4 weeks, how often was your child bothered by breathing problems, such as wheezing, coughing, or shortness of breath?

$\square \quad$ Not at all $=20$

- Once or twice $=15$

D Once every week $=10$

- 2 or 3 times a week $=5$

- 4 or more times a week $=0$

SCORE =

2. During the past 4 weeks, how often did your child's breathing problems (wheezing, coughing, shortness of breath) wake him or her up at night?

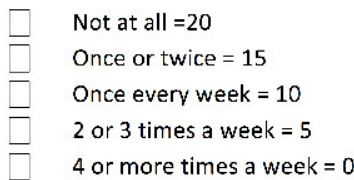

SCORE $=$

3. During the past 4 weeks, to what extent did your child's breathing problems, such as wheezing, coughing, or shortness of breath, interfere with his or her ability to play, go to school, or engage in usual activities that a child should be doing at his or her age?

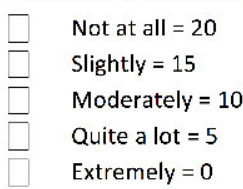

SCORE $=$

4. During the past 3 months, how often did you need to treat your child's breathing problems (wheezing, coughing, shortness of breath) with quick-relief medications (albuterol, Ventolin, Proventil, Maxair, ProAir, Xopenex, or Primatene Mist)?

- Not at all $=20$

Once or twice $=15$

Once every week $=10$

2 or 3 times a week $=5$

4 or more times a week $=0$

SCORE $=$

5. During the past 12 months, how often did your child need to take oral corticosteroids (prednisone, prednisolone, Orapred, Prelone, or Decadron) for breathing problems not controlled by other medications?

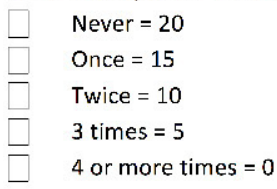

SCORE =

TOTAL SCORE =

Fig. 5 Test for Respiratory and Asthma Control in Kids. Caregiver self-report tool of asthma symptoms in children under 5 years of age. A score $<80$ indicates uncontrolled asthma. 


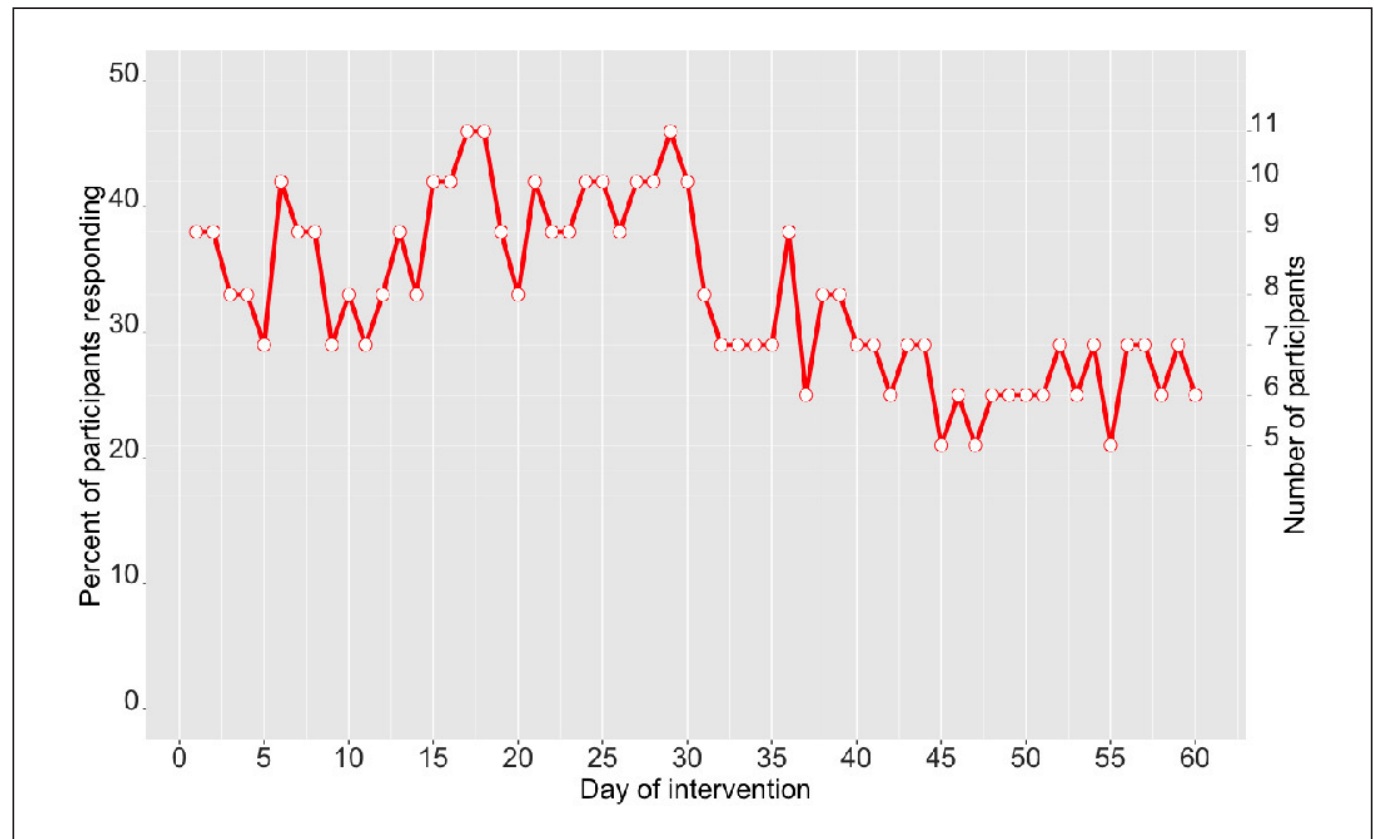

Fig. 6 Percent of Participants Responding to Text Messages. Percent of participants in intervention group that responded to text messages. The percent of respondents are on the left y-axis and the total number of participants responding is shown on the right $y$-axis for each day of the intervention.

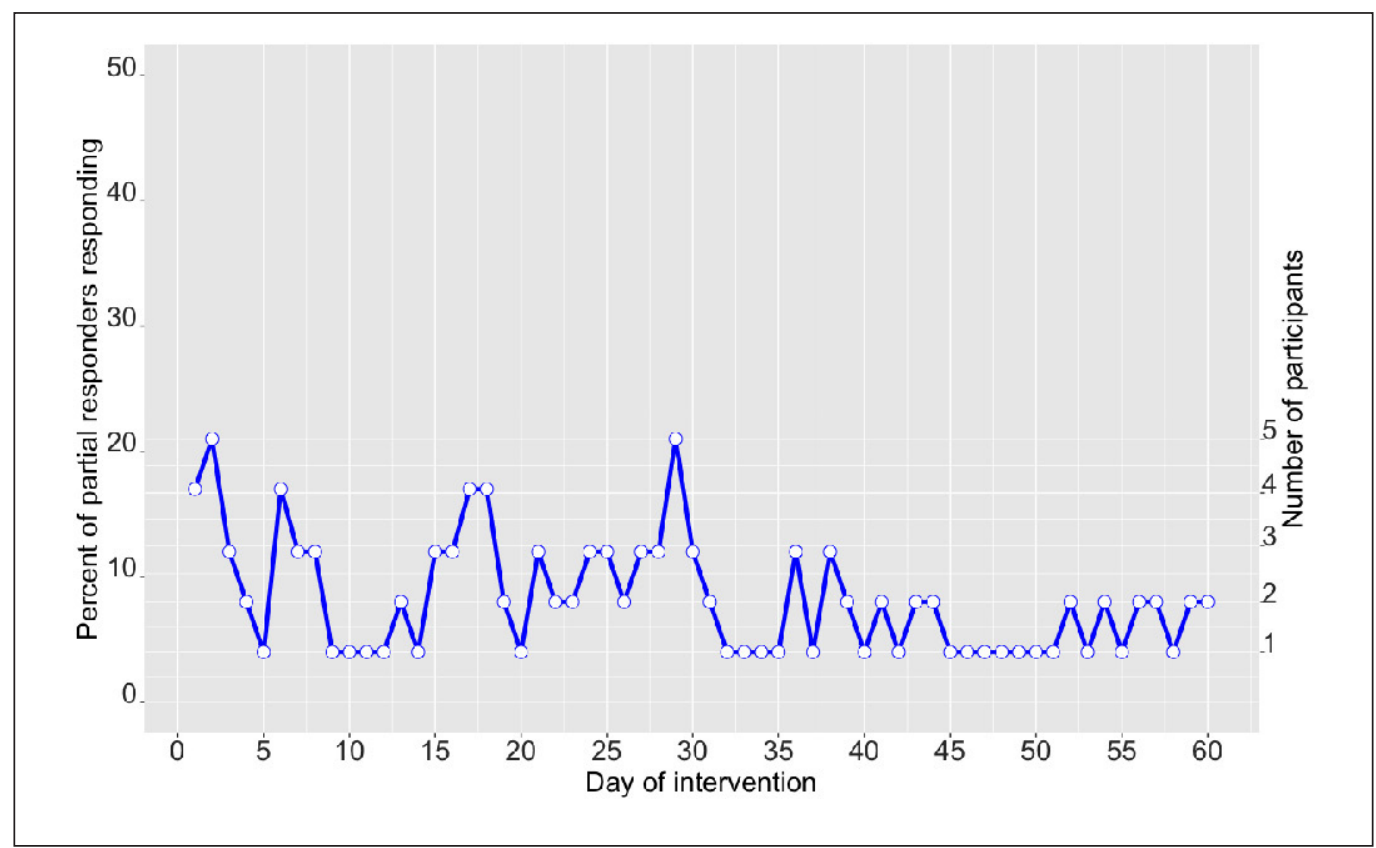

Fig. 7 Percent of Partial Responders Responding to Text Messages. Percent of partial responders (responded at least once, but less than $95 \%$ of the time) that responded to text messages. The percent of respondents are on the left $y$-axis and the total number of participants responding is shown on the right $y$-axis for each day of the intervention. 
Table 1 Baseline characteristics comparing the intervention (medication reminders) and control groups

\begin{tabular}{|l|l|l|l|l|}
\hline & $\begin{array}{l}\text { Total } \\
(\%) \mathbf{n}=47\end{array}$ & $\begin{array}{l}\text { Intervention group } \\
\mathbf{n}=26(\%)\end{array}$ & $\begin{array}{l}\text { Control group } \\
\mathbf{n}=21(\%)\end{array}$ & p value* \\
\hline Age, Median (IQR) & $20(9.5,25.5)$ & $20(11,25)$ & $20.5(8.75,25.5)$ & 0.741 \\
\hline Male & $24(51.1 \%)$ & $14(53.8 \%)$ & $10(47.6 \%)$ & 0.671 \\
\hline Race: African American & $46(98 \%)$ & $25(96 \%)$ & $21(100 \%)$ & 0.392 \\
\hline Adult & $29(61.7 \%)$ & $16(61.5 \%)$ & $13(61.9 \%)$ & 0.980 \\
\hline Asthma diagnosis & $27(57.4 \%)$ & $14(53.8 \%)$ & $13(61.9 \%)$ & 0.579 \\
\hline $\begin{array}{l}\text { Baseline medication adherence, } \\
\text { Mean (SD) (n=45) }\end{array}$ & $3.64(2.20)$ & $3.42(2.15)$ & $3.90(2.28)$ & 0.463 \\
\hline $\begin{array}{l}\text { Endpoint medication adherence, } \\
\text { Mean (SD) (n=40) }\end{array}$ & $5.18(1.88)$ & $5.46(1.74)$ & $4.75(2.05)$ & 0.248 \\
\hline Retention & $47(85.1 \%)$ & $24(92.3)$ & $16(76.2)$ & 0.217 \\
\hline
\end{tabular}

* Percentages compared with a chi-square test or a Fisher's exact test. Age compared using a Mann-Whitney U test. Morisky Medication Adherence Scale-8 scores compared using a t test.

$\mathrm{IQR}=$ Inter-Quartile Range; $\mathrm{SD}=$ Standard deviation

Table 2 Responses to text messages: All participants received reminder texts for their medications. If they responded with a 'no' a second reminder was sent an hour later.

\begin{tabular}{|l|l|l|l|l|}
\hline & $\begin{array}{l}\text { Number of } \\
\text { texts with re- } \\
\text { sponses }\end{array}$ & $\begin{array}{l}\text { 'No' re- } \\
\text { sponses }\end{array}$ & $\begin{array}{l}\text { Number of partici- } \\
\text { pants that texts } \\
\text { were sent to }\end{array}$ & $\begin{array}{l}\text { Number of par- } \\
\text { ticipants that re- } \\
\text { sponded }\end{array}$ \\
\hline $\begin{array}{l}\text { First medication } \\
\text { reminder text }\end{array}$ & 669 & 53 & 26 & 17 \\
\hline $\begin{array}{l}\text { Second medication } \\
\text { reminder text }\end{array}$ & 35 & 13 & 12 & 8 \\
\hline
\end{tabular}

Table 3 Changes in medication adherence scores within pediatric and adult patients, patients on hydroxyurea medication, and on asthma medication, mean (standard deviation).

\begin{tabular}{|l|l|l|l|}
\hline & $\begin{array}{l}\text { Intervention Group } \\
(\mathbf{n}=22)\end{array}$ & $\begin{array}{l}\text { Control Group } \\
(\mathbf{n}=16)\end{array}$ & p value* \\
\hline All participants & $1.91(2.35)$ & $0.94(2.05)$ & 0.118 \\
\hline Children $(\mathbf{n = 1 7 )}$ & $1.78(2.49)$ & $0.25(1.83)$ & 0.156 \\
\hline Adults $(\mathbf{n = 2 1 )}$ & $2.00(2.34)$ & $1.62(2.13)$ & 0.289 \\
\hline Hydroxyurea alone $(\mathbf{n = 3 1 )}$ & $2.06(2.34)$ & $1.31(2.06)$ & 0.230 \\
\hline Asthma medications alone $(\mathbf{n}=\mathbf{2 4})$ & $1.79(2.52)$ & $0.40(2.17)$ & 0.182 \\
\hline
\end{tabular}

* Changes in pre and post mean medication adherence scores between treatment groups were analyzed using a linear mixed model with time as a repeated factor and an autoregressive covariance matrix. Mixed models use all available data even when a patient has a missing score on either the pre or post medication adherence measure. 


\section{References}

1. Prevention CfDCa. Data \& Statistics 2015 [cited 2015 December 10]. Available from: http://www.cdc.gov/ ncbddd/sicklecell/data.html.

2. Fox S. Health Topics: @pewinternet; 2011 [cited 2015 6/14/2015]. Available from: http://www.pewinternet. org/2011/02/01/health-topics-2/.

3. Hamine S, Gerth-Guyette E, Faulx D, Green BB, Ginsburg AS. Impact of mHealth chronic disease management on treatment adherence and patient outcomes: a systematic review. J Med Internet Res 2015; 17(2): e52. doi: 10.2196/jmir.3951. PubMed PMID: 25803266; PubMed Central PMCID: PMCPMC4376208.

4. Jhamb M, Cavanaugh KL, Bian A, Chen G, Ikizler TA, Unruh ML, Abdel-Kader K. Disparities in electronic health record patient portal use in nephrology clinics. Clinical Journal of the American Society of Nephrology 2015; 10(11): 2013-2022.

5. Osborn CY, Mayberry LS, Wallston KA, Johnson KB, Elasy TA. Understanding patient portal use: implications for medication management. J Med Internet Res 2013; 15(7): e133. doi: 10.2196/jmir.2589. PubMed PMID: 23823974; PubMed Central PMCID: PMCPMC3713921.

6. Smith SG, O`Conor R, Aitken W, Curtis LM, Wolf MS, Goel MS. Disparities in registration and use of an online patient portal among older adults: findings from the LitCog cohort. J Am Med Inform Assoc 2015; 22(4): 888-895. doi: 10.1093/jamia/ocv025. PubMed PMID: 25914099; PubMed Central PMCID: PMCPMC4810779.

7. Nelson LA, Mulvaney SA, Gebretsadik T, Ho YX, Johnson KB, Osborn CY. Disparities in the use of a mHealth medication adherence promotion intervention for low-income adults with type 2 diabetes. J Am Med Inform Assoc 2016; 23(1): 12-18. doi: 10.1093/jamia/ocv082. PubMed PMID: 26186935; PubMed Central PMCID: PMCPMC5009937.

8. Charache S, Terrin ML, Moore RD, Dover GJ, Barton FB, Eckert SV, McMahon RP, Bonds DR. Effect of hydroxyurea on the frequency of painful crises in sickle cell anemia. Investigators of the Multicenter Study of Hydroxyurea in Sickle Cell Anemia. N Engl J Med 1995; 332(20): 1317-1322. doi: 10.1056/NEJM199505183322001. PubMed PMID: 7715639.

9. Kinney TR, Helms RW, O'Branski EE, Ohene-Frempong K, Wang W, Daeschner C, Vichinsky E, ReddingLallinger R, Gee B, Platt OS, Ware RE. Safety of hydroxyurea in children with sickle cell anemia: results of the HUG-KIDS study, a phase I/II trial. Pediatric Hydroxyurea Group. Blood 1999; 94(5): 1550-1554. PubMed PMID: 10477679.

10. Wang WC, Ware RE, Miller ST, Iyer RV, Casella JF, Minniti CP, Rana S, Thornburg CD, Rogers ZR, Kalpatthi RV, Barredo JC, Brown RC, Sarnaik SA, Howard TH, Wynn LW, Kutlar A, Armstrong FD, Files BA, Goldsmith JC, Waclawiw MA, Huang X, Thompson BW, investigators BH. Hydroxycarbamide in very young children with sickle-cell anaemia: a multicentre, randomised, controlled trial (BABY HUG). Lancet 2011; 377(9778): 1663-1672. doi: 10.1016/S0140-6736(11)60355-3. PubMed PMID: 21571150; PubMed Central PMCID: PMCPMC3133619.

11. Anim SO, Strunk RC, DeBaun MR. Asthma morbidity and treatment in children with sickle cell disease. Expert Rev Respir Med. 2011;5(5):635-45. doi: 10.1586/ers.11.64. PubMed PMID: 21955234; PubMed Central PMCID: PMCPMC3233260.

12. Newaskar M, Hardy KA, Morris CR. Asthma in sickle cell disease. Scientific World Journal 2011; 11: 1138-1152. doi: 10.1100/tsw.2011.105. PubMed PMID: 21623460.

13. National Asthma E, Prevention P. Expert Panel Report 3 (EPR-3): Guidelines for the Diagnosis and Management of Asthma-Summary Report 2007. J Allergy Clin Immunol 2007; 120(5 Suppl): S94-S138. doi: 10.1016/j.jaci.2007.09.043. PubMed PMID: 17983880.

14. Candrilli SD, O'Brien SH, Ware RE, Nahata MC, Seiber EE, Balkrishnan R. Hydroxyurea adherence and associated outcomes among Medicaid enrollees with sickle cell disease. Am J Hematol 2011; 86(3): 273-277. doi: 10.1002/ajh.21968. PubMed PMID: 21328441.

15. DiMatteo MR. Variations in patients' adherence to medical recommendations: a quantitative review of 50 years of research. Med Care 2004; 42(3): 200-209. PubMed PMID: 15076819.

16. Thornburg CD, Calatroni A, Telen M, Kemper AR. Adherence to hydroxyurea therapy in children with sickle cell anemia. J Pediatr 2010; 156(3): 415-419. doi: 10.1016/j.jpeds.2009.09.044. PubMed PMID: 19880135; PubMed Central PMCID: PMCPMC3901082.

17. Walsh KE, Cutrona SL, Kavanagh PL, Crosby LE, Malone C, Lobner K, Bundy DG. Medication adherence among pediatric patients with sickle cell disease: a systematic review. Pediatrics 2014; 134(6): 1175-1183. doi: 10.1542/peds.2014-0177. PubMed PMID: 25404717; PubMed Central PMCID: PMCPMC4243064. 
18. Badawy SM, Thompson AA, Lai JS, Penedo FJ, Rychlik K, Liem RI. Health-related quality of life and adherence to hydroxyurea in adolescents and young adults with sickle cell disease. Pediatr Blood Cancer 2016. doi: 10.1002/pbc.26369. PubMed PMID: 27896936.

19. Johnson KB, Patterson BL, Ho YX, Chen Q, Nian H, Davison CL, Slagle J, Mulvaney SA. The feasibility of text reminders to improve medication adherence in adolescents with asthma. J Am Med Inform Assoc 2015. doi: 10.1093/jamia/ocv158. PubMed PMID: 26661717.

20. Jhamb M, Cavanaugh KL, Bian A, Chen G, Ikizler TA, Unruh ML, Abdel-Kader K. Disparities in Electronic Health Record Patient Portal Use in Nephrology Clinics. Clin J Am Soc Nephrol 2015; 10(11): 2013-2022. doi: 10.2215/CJN.01640215. PubMed PMID: 26493242; PubMed Central PMCID: PMCPMC4633780.

21. Anderson C, Torres S, Gokuli S, Brandow AM, Panepinto JA. Assessment of communication modes in patients and families with sickle cell disease. Pediatr Blood Cancer 2013; 60(5): 887. doi: 10.1002/pbc.24477. PubMed PMID: 23450782.

22. Badawy SM, Thompson AA, Liem RI. Technology Access and Smartphone App Preferences for Medication Adherence in Adolescents and Young Adults With Sickle Cell Disease. Pediatr Blood Cancer 2016. Epub 2016/02/05. doi: 10.1002/pbc.25905. PubMed PMID: 26844685.

23.Lenhart A. Teens, social media \& technology overview 2015. Pew Research Center 2015; 9.

24. Smith A. US smartphone use in 2015. Pew Research Center 2015; 1.

25. Thakkar J, Kurup R, Laba TL, Santo K, Thiagalingam A, Rodgers A, Woodward M, Redfern J, Chow CK. Mobile Telephone Text Messaging for Medication Adherence in Chronic Disease: A Meta-analysis. JAMA Intern Med 2016; 176(3): 340-349. doi: 10.1001/jamainternmed.2015.7667. PubMed PMID: 26831740.

26. Estepp JH, Winter B, Johnson M, Smeltzer MP, Howard SC, Hankins JS. Improved hydroxyurea effect with the use of text messaging in children with sickle cell anemia. Pediatr Blood Cancer 2014; 61(11): 2031-2036. Epub 2014/08/19. doi: 10.1002/pbc.25177. PubMed PMID: 25132074.

27. Wald DS, Butt S, Bestwick JP. One-way versus two-way text messaging on improving medication adherence: meta-analysis of randomized trials. Am J Med 2015; 128(10): 1139 e1-5. doi: 10.1016/j.amjmed.2015.05.035. PubMed PMID: 26087045.

28. QuickCalcs GS. Randomly assign subjects to treatment groups 2016 [cited 2016]. Available from: http://www.graphpad.com/quickcalcs/randomize1.cfm.

29. Harris PA, Taylor R, Thielke R, Payne J, Gonzalez N, Conde JG. Research electronic data capture (REDCap)--a metadata-driven methodology and workflow process for providing translational research informatics support. J Biomed Inform 2009; 42(2): 377-381. doi: 10.1016/j.jbi.2008.08.010. PubMed PMID: 18929686; PubMed Central PMCID: PMCPMC2700030.

30. Tickle-Degnen L. Nuts and bolts of conducting feasibility studies. The American journal of occupational therapy : official publication of the American Occupational Therapy Association 2013; 67(2): 171-176. doi: 10.5014/ajot.2013.006270. PubMed PMID: 23433271; PubMed Central PMCID: PMCPMC3722658.

31. Morisky DE, Ang A, Krousel区Wood M, Ward HJ. Predictive validity of a medication adherence measure in an outpatient setting. The Journal of Clinical Hypertension 2008; 10(5): 348-354.

32. Krousel-Wood M, Islam T, Webber LS, Re RN, Morisky DE, Muntner P. New medication adherence scale versus pharmacy fill rates in seniors with hypertension. Am J Manag Care 2009; 15(1): 59-66. Epub 2009/01/17. PubMed PMID: 19146365; PubMed Central PMCID: PMCPMC2728593.

33. Trindade AJ, Ehrlich A, Kornbluth A, Ullman TA. Are your patients taking their medicine? Validation of a new adherence scale in patients with inflammatory bowel disease and comparison with physician perception of adherence. Inflamm Bowel Dis 2011; 17(2): 599-604. doi: 10.1002/ibd.21310. PubMed PMID: 20848512.

34. Schatz M, Sorkness CA, Li JT, Marcus P, Murray JJ, Nathan RA, Kosinski M, Pendergraft TB, Jhingran P. Asthma Control Test: reliability, validity, and responsiveness in patients not previously followed by asthma specialists. J Allergy Clin Immunol 2006; 117(3): 549-556. doi: 10.1016/j.jaci.2006.01.011. PubMed PMID: 16522452.

35. Voorend-van Bergen S, Vaessen-Verberne AA, Landstra AM, Brackel HJ, van den Berg NJ, Caudri D, de Jongste JC, Merkus PJ, Pijnenburg MW. Monitoring childhood asthma: web-based diaries and the asthma control test. J Allergy Clin Immunol 2014; 133(6): 1599-1605 e2. doi: 10.1016/j.jaci.2013.10.005. PubMed PMID: 24290276.

36.Schatz M, Kosinski M, Yarlas AS, Hanlon J, Watson ME, Jhingran P. The minimally important difference of the Asthma Control Test. J Allergy Clin Immunol 2009; 124(4): 719-723 e1. doi: 10.1016/j.jaci.2009.06.053. PubMed PMID: 19767070.

37.Zeiger RS, Mellon M, Chipps B, Murphy KR, Schatz M, Kosinski M, Lampl K, Ramachandran S. Test for Respiratory and Asthma Control in Kids (TRACK): clinically meaningful changes in score. J Allergy Clin Immunol 2011; 128(5): 983-988. doi: 10.1016/j.jaci.2011.08.010. PubMed PMID: 21906790. 
38. Hardy H, Kumar V, Doros G, Farmer E, Drainoni ML, Rybin D, Myung D, Jackson J, Backman E, Stanic A, Skolnik PR. Randomized controlled trial of a personalized cellular phone reminder system to enhance adherence to antiretroviral therapy. AIDS Patient Care STDS 2011; 25(3): 153-161. doi: 10.1089/apc.2010.0006. PubMed PMID: 21323532; PubMed Central PMCID: PMCPMC3101947.

39. Park LG, Howie-Esquivel J, Chung ML, Dracup K. A text messaging intervention to promote medication adherence for patients with coronary heart disease: a randomized controlled trial. Patient Educ Couns 2014; 94(2): 261-268. doi: 10.1016/j.pec.2013.10.027. PubMed PMID: 24321403.

40. Wald DS, Bestwick JP, Raiman L, Brendell R, Wald NJ. Randomised trial of text messaging on adherence to cardiovascular preventive treatment (INTERACT trial). PLoS ONE 2014; 9(12): e114268. doi: 10.1371/journal.pone.0114268. PubMed PMID: 25479285; PubMed Central PMCID: PMCPMC4257733.

41.Lv Y, Zhao H, Liang Z, Dong H, Liu L, Zhang D, Cai S. A mobile phone short message service improves perceived control of asthma: a randomized controlled trial. Telemed J E Health 2012; 18(6): 420-426. doi: 10.1089/tmj.2011.0218. PubMed PMID: 22667695.

42.Strandbygaard U, Thomsen SF, Backer V. A daily SMS reminder increases adherence to asthma treatment: a three-month follow-up study. Respir Med 2010; 104(2): 166-171. doi: 10.1016/j.rmed.2009.10.003. PubMed PMID: 19854632.

43. Kannisto KA, Koivunen MH, Valimaki MA. Use of mobile phone text message reminders in health care services: a narrative literature review. J Med Internet Res 2014; 16(10): e222. doi: 10.2196/jmir.3442. PubMed PMID: 25326646; PubMed Central PMCID: PMCPMC4211035.

44. da Costa TM, Salomao PL, Martha AS, Pisa IT, Sigulem D. The impact of short message service text messages sent as appointment reminders to patients' cell phones at outpatient clinics in Sao Paulo, Brazil. Int J Med Inform 2010; 79(1): 65-70. doi: 10.1016/j.ijmedinf.2009.09.001. PubMed PMID: 19783204.

45.Leong KC, Chen WS, Leong KW, Mastura I, Mimi O, Sheikh MA, Zailinawati AH, Ng CJ, Phua KL, Teng CL. The use of text messaging to improve attendance in primary care: a randomized controlled trial. Fam Pract 2006; 23(6): 699-705. doi: 10.1093/fampra/cml044. PubMed PMID: 16916871.

46. Wang K, Wang C, Xi L, Zhang Y, Ouyang Y, Lou H, Zhang W, Zhang L. A randomized controlled trial to assess adherence to allergic rhinitis treatment following a daily short message service (SMS) via the mobile phone. Int Arch Allergy Immunol 2014; 163(1): 51-58. doi: 10.1159/000356317. PubMed PMID: 24248037. 